\title{
Applications of Discrete Convex Analysis to Mathematical Economics ${ }^{\dagger}$
}

By

\author{
Akihisa TAMURA*
}

\begin{abstract}
Discrete convex analysis, which is a unified framework of discrete optimization, is being recognized as a basic tool for mathematical economics. This paper surveys the recent progress in applications of discrete convex analysis to mathematical economics.
\end{abstract}

\section{$\S 1$. Introduction}

Discrete convex analysis, proposed by Murota [25, 26], is a unified framework of discrete optimization. Recently, applications of discrete convex analysis to mathematical economics have been investigated. The aim of this paper is to survey the following recent progress on this topic.

The concepts of M-convex functions due to Murota [25, 26] and $\mathrm{M}^{\natural}$-convex functions due to Murota and Shioura [30], which play central roles in discrete convex analysis, are being recognized as nice discrete convex functions from the point of view of mathematical economics. For instance, for set functions, Fujishige and Yang [15] showed that $\mathrm{M}^{\natural}$-concavity is equivalent to the gross substitutability and the single improvement property which are equivalent to each other for set functions [17] and are nice in the following sense. These properties guarantee the existence of the core of several models, e.g., a matching model proposed by Kelso and Crawford [21]. Relations among these three properties were extended to the general case by Danilov, Koshevoy and Lang [3] and by

Communicated by S. Fujishige. Received January 29, 2004.

2000 Mathematics Subject Classification(s): 90C25, 91B50.

Supported by a Grant-in-Aid for Scientific Research from the Ministry of Education, Culture, Sports, Science and Technology of Japan.

${ }^{\dagger}$ This article is an invited contribution to a special issue of Publications of RIMS commemorating the fortieth anniversary of the founding of the Research Institute for Mathematical Sciences.

*RIMS, Kyoto University, Kyoto 606-8502, Japan.

e-mail: tamura@kurims.kyoto-u.ac.jp 
Murota and Tamura [33]. Furthermore, Farooq and Tamura [9] characterized $\mathrm{M}^{\natural}$-concave set functions by using the substitutability which guarantees the existence of a stable matching of generalized stable marriage models due to Roth [35, 36], Sotomayor [41], Alkan and Gale [1] and Fleiner [11].

On the other hand, economic models based on $\mathrm{M}^{\natural}$-concave utility functions have been proposed. Danilov, Koshevoy and Murota [5] provided a model based on discrete convex analysis and showed the existence of a competitive equilibrium in an exchange economy with indivisible commodities when the utility function of each agent is quasilinear in money and its reservation value function is $\mathrm{M}^{\natural}$-concave. Murota and Tamura [34] proposed an efficient algorithm for finding a competitive equilibrium of the Danilov-Koshevoy-Murota model. Danilov, Koshevoy and Lang [4] showed the existence of a competitive equilibrium in a model in which commodities are partitioned into two groups: substitutes and complements. B. Lehmann, D. Lehmann and Nisan [22] discussed a combinatorial auction with $\mathrm{M}^{\natural}$-concave utilities. Eguchi and Fujishige [6] extended the stable marriage model to the framework of discrete convex analysis. Eguchi, Fujishige and Tamura [7] extended the Eguchi-Fujishige model so that indifference on preferences and multiple partnerships are allowed. Fujishige and Tamura [14] proposed a common generalization of the stable marriage model and the assignment model by utilizing $\mathrm{M}^{\natural}$-concave utilities and verified the existence of a stable solution of their general model.

The present paper is organized as follows. Section 2 briefly introduces known results on $\mathrm{M}-/ \mathrm{M}^{\natural}$-concave functions. Section 3 discusses relations among the gross substitutability, the single improvement property, the substitutability and $\mathrm{M}^{\natural}$-concavity. Section 4 overviews two-sided matching market models. Sections 5, 6, 7, 8 and 9 explain the above-mentioned economic models based on discrete convex analysis. In Section 10, we give several open problems.

\section{§2. $\quad M-/ M^{\natural}-$ Concavity}

Since a utility function is usually assumed to be concave in mathematical economics, we review several definitions and known results on M-concave functions and $\mathrm{M}^{\natural}$-concave functions.

Let $V$ be a nonempty finite set, and let $\mathbf{Z}$ and $\mathbf{R}$ be the sets of integers and reals, respectively. We denote by $\mathbf{Z}^{V}$ the set of integral vectors $x=(x(v): v \in$ $V$ ) indexed by $V$, where $x(v)$ denotes the $v$ th component of vector $x$. Also, $\mathbf{R}^{V}$ denotes the set of real vectors indexed by $V$. We define the positive support and negative support of $z=(z(v): v \in V) \in \mathbf{Z}^{V}$ by

$$
\operatorname{supp}^{+}(z)=\{v \in V \mid z(v)>0\} \quad \text { and } \operatorname{supp}^{-}(z)=\{v \in V \mid z(v)<0\} .
$$


For each $S \subseteq V$, we denote by $\chi_{S}$ the characteristic vector of $S$ defined by $\chi_{S}(v)=1$ if $v \in S$; otherwise $\chi_{S}(v)=0$, and write simply $\chi_{u}$ instead of $\chi_{\{u\}}$ for each $u \in V$. For a vector $p \in \mathbf{R}^{V}$ and a function $f: \mathbf{Z}^{V} \rightarrow \mathbf{R} \cup\{ \pm \infty\}$, we define functions $\langle p, x\rangle$ and $f[p](x)$ by

$$
\langle p, x\rangle=\sum_{v \in V} p(v) x(v) \quad \text { and } \quad f[p](x)=f(x)+\langle p, x\rangle
$$

for all $x \in \mathbf{Z}^{V}$ and define the set of maximizers of $f$ and the effective domain of $f$ by

$$
\begin{aligned}
\arg \max f & =\left\{x \in \mathbf{Z}^{V} \mid f(x) \geq f(y) \quad\left(\forall y \in \mathbf{Z}^{V}\right)\right\}, \\
\operatorname{dom} f & =\left\{x \in \mathbf{Z}^{V} \mid-\infty<f(x)<+\infty\right\} .
\end{aligned}
$$

A function $f: \mathbf{Z}^{V} \rightarrow \mathbf{R} \cup\{-\infty\}$ with $\operatorname{dom} f \neq \emptyset$ is called $M$-concave $[25,26]$ if it satisfies

$(-\mathrm{M}-\mathrm{EXC})$ for all $x, y \in \operatorname{dom} f$ and all $u \in \operatorname{supp}^{+}(x-y)$, there exists $v \in$ $\operatorname{supp}^{-}(x-y)$ such that

$$
f(x)+f(y) \leq f\left(x-\chi_{u}+\chi_{v}\right)+f\left(y+\chi_{u}-\chi_{v}\right) .
$$

From (-M-EXC), the effective domain of an M-concave function lies on a hyperplane $\left\{x \in \mathbf{R}^{V} \mid x(V)=\right.$ constant $\}$, where $x(V)=\sum_{v \in V} x(v)$.

The concept of $\mathrm{M}^{\natural}$-concavity is a variant of M-concavity. Let 0 denote a new element not in $V$ and define $\hat{V}=\{0\} \cup V$. A function $f: \mathbf{Z}^{V} \rightarrow \mathbf{R} \cup\{-\infty\}$ with $\operatorname{dom} f \neq \emptyset$ is called $M^{\natural}$-concave [30] if it is expressed in terms of an Mconcave function $\hat{f}: \mathbf{Z}^{\hat{V}} \rightarrow \mathbf{R} \cup\{-\infty\}$ as: for all $x \in \mathbf{Z}^{V}$

$$
f(x)=\hat{f}\left(x_{0}, x\right) \quad \text { with } x_{0}=-x(V) .
$$

Namely, an $\mathrm{M}^{\natural}$-concave function is a function obtained as the projection of an M-concave function. Conversely, an $\mathrm{M}^{\natural}$-concave function $f$ determines the corresponding M-concave function $\hat{f}$ by

$$
\hat{f}\left(x_{0}, x\right)= \begin{cases}f(x) & \text { if } x_{0}=-x(V) \\ -\infty & \text { otherwise }\end{cases}
$$

for all $\left(x_{0}, x\right) \in \mathbf{Z}^{\hat{V}}$. An $\mathrm{M}^{\natural}$-concave function can also be defined by using an exchange property.

Theorem 2.1 [30]. A function $f: \mathbf{Z}^{V} \rightarrow \mathbf{R} \cup\{-\infty\}$ with $\operatorname{dom} f \neq \emptyset$ is $M^{\natural}$-concave if and only if it satisfies 
$\left(-\mathrm{M}^{\natural}-\mathrm{EXC}\right)$ for all $x, y \in \operatorname{dom} f$ and all $u \in \operatorname{supp}^{+}(x-y)$, there exists $v \in$ $\{0\} \cup \operatorname{supp}^{-}(x-y)$ such that

$$
f(x)+f(y) \leq f\left(x-\chi_{u}+\chi_{v}\right)+f\left(y+\chi_{u}-\chi_{v}\right),
$$

where we assume $\chi_{0}$ is the zero vector on $V$.

Whereas the concept of $\mathrm{M}^{\natural}$-concavity is equivalent to that of M-concavity as above, Theorem 2.1 and the definition of M-concavity imply that an Mconcave function is $\mathrm{M}^{\natural}$-concave. That is, we have

$$
f:\left(-\mathrm{M}^{\natural}-\mathrm{EXC}\right) \Longleftrightarrow \hat{f}:(-\mathrm{M}-\mathrm{EXC}) \Longrightarrow \hat{f}:\left(-\mathrm{M}^{\natural}-\mathrm{EXC}\right) .
$$

In the sequel, we concentrate an $\mathrm{M}^{\natural}$-concave function and assume that its function value for each point can be calculated in constant time.

The maximizers of an $\mathrm{M}^{\natural}$-concave function has a good characterization.

Theorem $2.2[25,26]$. For an $M^{\natural}$-concave function $f: \mathbf{Z}^{V} \rightarrow \mathbf{R} \cup$ $\{-\infty\}$ and $x \in \operatorname{dom} f, x \in \arg \max f$ if and only if $f(x) \geq f\left(x-\chi_{u}+\chi_{v}\right)$ for all $u, v \in\{0\} \cup V$.

Theorem 2.2 says that we can check whether a given point $x$ is a maximizer of $f$ or not in $\mathrm{O}\left(|V|^{2}\right)$ time. Furthermore, it is known that a problem of maximizing an $\mathrm{M}^{\natural}$-concave function $f$ can be solved in polynomial time in $|V|$ and $\log L$, where $L=\max \left\{\|x-y\|_{\infty} \mid x, y \in \operatorname{dom} f\right\}$ (see $[45,40]$ ).

The sum of two $\mathrm{M}^{\natural}$-concave functions is not $\mathrm{M}^{\natural}$-concave in general. So we need a sophisticated characterization for the maximizers of the sum of two $\mathrm{M}^{\natural}$-concave functions.

Theorem 2.3 [25]. For $M^{\natural}$-concave functions $f_{1}, f_{2}: \mathbf{Z}^{V} \rightarrow \mathbf{R} \cup\{-\infty\}$ and a point $x^{*} \in \operatorname{dom} f_{1} \cap \operatorname{dom} f_{2}$, we have $x^{*} \in \arg \max \left(f_{1}+f_{2}\right)$ if and only if there exists $p^{*} \in \mathbf{R}^{V}$ such that $x^{*} \in \arg \max f_{1}\left[+p^{*}\right]$ and $x^{*} \in \arg \max f_{2}\left[-p^{*}\right]$, and furthermore, for such $p^{*}$, we have

$$
\arg \max \left(f_{1}+f_{2}\right)=\arg \max \left(f_{1}\left[+p^{*}\right]\right) \cap \arg \max \left(f_{2}\left[-p^{*}\right]\right) .
$$

We call the problem of maximizing the sum of two $\mathrm{M}^{\natural}$-concave functions the $\mathrm{M}^{\natural}$-concave intersection problem. It is known that the $\mathrm{M}^{\natural}$-concave intersection problem for integer-valued $\mathrm{M}^{\natural}$-concave functions can be solved in polynomial time (see $[19,18]$ ).

The integer convolution $f$ of a finite family $\left\{f_{i} \mid i \in I\right\}$ of $\mathrm{M}^{\natural}$-concave functions defined by

$$
f(x)=\sup \left\{\sum_{i \in I} f_{i}\left(x_{i}\right) \mid \sum_{i \in I} x_{i}=x, x_{i} \in \mathbf{Z}^{V}(\forall i \in I)\right\}
$$


for all $x \in \mathbf{Z}^{V}$ is also $\mathrm{M}^{\natural}$-concave [25]. It is known that the problem of calculating $f(x)$ for a given $x$ is transformed to the $\mathrm{M}^{\natural}$-concave intersection problem (e.g., see [29]).

\section{$\S 3 . \quad M^{\natural}$-Concavity in Mathematical Economics}

In this section, we describe nice features of an $\mathrm{M}^{\natural}$-concave function as a utility function from the point of view of mathematical economics.

For a function $f: \mathbf{Z}^{V} \rightarrow \mathbf{R} \cup\{-\infty\}$, its concave closure $\underline{f}$ is defined by

$$
\underline{f}(z)=\inf _{p \in \mathbf{R}^{V}, \alpha \in \mathbf{R}}\left\{\langle p, z\rangle+\alpha \mid\langle p, y\rangle+\alpha \geq f(y)\left(\forall y \in \mathbf{Z}^{V}\right)\right\}
$$

for all $z \in \mathbf{Z}^{V}$. We say that $f$ is concave-extensible if $f(x)=\underline{f}(x)$ for all $x \in \mathbf{Z}^{V}$. An $\mathrm{M}^{\natural}$-concave function deserves its name in the following sense.

Lemma 3.1 [25]. An $M^{\natural}$-concave function is concave-extensible.

A utility function generally has decreasing marginal returns, which is equivalent to submodularity in the binary case. This is also the case with an $\mathrm{M}^{\natural}$-concave function.

Lemma 3.2 [32]. An $M^{\natural}$-concave function $f$ is submodular, i.e.,

$$
f(x)+f(y) \geq f(x \vee y)+f(x \wedge y)
$$

for all $x, y \in \operatorname{dom} f$, where vectors $x \vee y$ and $x \wedge y$ are defined by: for all $v \in V$

$$
(x \vee y)(v)=\max \{x(v), y(v)\}, \quad(x \wedge y)(v)=\min \{x(v), y(v)\} .
$$

We now consider natural generalizations of the gross substitutability and the single improvement property which were originally proposed for set functions by Kelso and Crawford [21] and Gul and Stacchetti [17], respectively.

$\left(-\mathrm{GS}_{\mathrm{W}}\right)$ For all $p, q \in \mathbf{R}^{V}$ and all $x \in \operatorname{dom} f$ such that $p \leq q, x \in \arg \max f[-p]$ and $\arg \max f[-q] \neq \emptyset$, there exists $y \in \arg \max f[-q]$ such that $y(v) \geq$ $x(v)$ for all $v$ with $p(v)=q(v)$.

(-GS) For all $\left(p_{0}, p\right),\left(q_{0}, q\right) \in \mathbf{R}^{\{0\} \cup V}$ and all $x \in \operatorname{dom} f$ such that $\left(p_{0}, p\right) \leq$ $\left(q_{0}, q\right), x \in \arg \max f\left[-p+p_{0} \mathbf{1}\right]$ and $\arg \max f\left[-q+q_{0} \mathbf{1}\right] \neq \emptyset$, there exists $y \in \arg \max f\left[-q+q_{0} \mathbf{1}\right]$ such that $y(v) \geq x(v)$ for all $v$ with $p(v)=q(v)$ and $y(V) \leq x(V)$ if $p_{0}=q_{0}$, where $\mathbf{1}$ denotes the vector of all ones. 
(-SWGS) For all $p \in \mathbf{R}^{V}$, all $x \in \arg \max f[-p]$ and all $v \in V$, one of the following statements holds:

(i) $x \in \arg \max f\left[-p-\alpha \chi_{v}\right]$ for all $\alpha>0$,

(ii) there exist $\alpha>0$ and $y \in \arg \max f\left[-p-\alpha \chi_{v}\right]$ such that $y(v)=x(v)-1$ and $y(u) \geq x(u)$ for all $u \in V \backslash\{v\}$.

$\left(-\mathrm{SI}_{\mathrm{W}}\right)$ For all $p \in \mathbf{R}^{V}$ and all $x \in \operatorname{dom} f$ with $x \notin \arg \max f[-p]$, there exist $u, v \in\{0\} \cup V$ such that $f[-p](x)<f[-p]\left(x-\chi_{u}+\chi_{v}\right)$.

(-SI) For all $p \in \mathbf{R}^{V}$ and all $x, y \in \operatorname{dom} f$ with $f[-p](x)<f[-p](y)$,

$$
f[-p](x)<\max _{u \in\{0\} \cup \operatorname{supp}^{+}(x-y)} \max _{v \in\{0\} \cup \operatorname{supp}^{-}(x-y)} f[-p]\left(x-\chi_{u}+\chi_{v}\right) .
$$

We are primarily interested in the case where $\operatorname{dom} f$ is bounded. Then, for any $p \in \mathbf{R}^{V}$, arg max $f[-p]$ is nonempty. The above properties are interpreted as follows. Here, we assume that $V$ denotes the set of indivisible commodities, $x \in$ $\mathbf{Z}^{V}$ the numbers $x(v)$ of commodities $v$ consumed by a consumer and $f$ a utility function of the consumer. Property $\left(-\mathrm{GS}_{\mathrm{W}}\right)$ states that if the price vector is increased from $p$ to $q$ then the consumer still wants to consume at least the same numbers of the commodities whose prices remain the same. (-GS) says that when prices increase $\left(p \leq q\right.$ and $\left.p_{0}=q_{0}\right)$, the consumer wants a consumption such that the numbers of the commodities whose prices remain the same do not decrease and the total number of commodities does not increase. (-GS) also says that when all prices decrease by the same amount $\left(p=q\right.$ and $\left.p_{0}<q_{0}\right)$, the consumer wants at least the same number of each commodity. Obviously, the property $(-\mathrm{GS})$ is stronger than $\left(-\mathrm{GS}_{\mathrm{W}}\right)$. (-SWGS) is interpreted as follows: when the price of commodity $v$ is slightly increased, the consumer wants either (i) the same consumption or (ii) a consumption in which the number of commodity $v$ is decreased by exactly one and the numbers of the other commodities are not decreased. $\left(-\mathrm{SI}_{\mathrm{W}}\right)$ states that a nonoptimal consumption $x$ can be improved strictly by either removing a commodity from $x$, adding a commodity to $x$, or doing both. Moreover, $(-\mathrm{SI})$ is stronger than $\left(-\mathrm{SI}_{\mathrm{W}}\right)$ in the sense that the consumer can bring consumption $x$ nearer to any better consumption $y$.

Properties $\left(-\mathrm{GS}_{\mathrm{W}}\right)$ and $\left(-\mathrm{SI}_{\mathrm{W}}\right)$ for functions on $\{0,1\}$-hypercubes correspond to the gross substitutability and the single improvement property for set functions, respectively. We note that the equivalence between the gross substitutability and the single improvement property for set functions was first pointed out by Gul and Stacchetti [17], and that the equivalence between the 
single improvement property and $\mathrm{M}^{\natural}$-concavity for set functions was by $\mathrm{Fu}$ jishige and Yang [15]. (-SWGS) was given by Danilov, Koshevoy and Lang [3] and the other properties by Murota and Tamura [33]. An $\mathrm{M}^{\natural}$-concave function satisfies these properties, and furthermore, it is characterized by these properties.

Theorem 3.1 [33]. For a concave-extensible function $f: \mathbf{Z}^{V} \rightarrow \mathbf{R} \cup$ $\{-\infty\}$ with a bounded nonempty effective domain, $f$ is $M^{\natural}$-concave if and only if it satisfies (-GS).

Theorem 3.2 [3]. For a concave-extensible function $f: \mathbf{Z}^{V} \rightarrow \mathbf{R} \cup$ $\{-\infty\}$ with a nonempty effective domain, $f$ is $M^{\natural}$-concave if and only if it satisfies (-SWGS).

Theorem 3.3 [33]. For a function $f: \mathbf{Z}^{V} \rightarrow \mathbf{R} \cup\{-\infty\}$ with a nonempty effective domain, $f$ is $M^{\natural}$-concave if and only if it satisfies (-SI).

For a set function, we have the following result.

Theorem 3.4 [33]. For $f:\{0,1\}^{V} \rightarrow \mathbf{R} \cup\{-\infty\}$ with a nonempty effective domain ${ }^{1}$, we have

$$
\left(-\mathrm{M}^{\natural}-\mathrm{EXC}\right) \Leftrightarrow(-\mathrm{SI}) \Leftrightarrow\left(-\mathrm{SI}_{\mathrm{W}}\right) \Leftrightarrow(-\mathrm{GS}) \Leftrightarrow\left(-\mathrm{GS}_{\mathrm{W}}\right) .
$$

We next discuss relations between the substitutability and $\mathrm{M}^{\natural}$-concavity. Eguchi, Fujishige and Tamura [7] and Fujishige and Tamura [14] showed that $\mathrm{M}^{\natural}$-concavity implies the substitutability in the following sense.

Lemma $3.3[7,14] . \quad$ An $M^{\natural}$-concave function satisfies the following properties.

$\left(-\mathrm{SC}^{1}\right)$ For any $z_{1}, z_{2} \in \mathbf{Z}^{V}$ such that $z_{1} \geq z_{2}$ and $\arg \max \left\{f(y) \mid y \leq z_{2}\right\} \neq \emptyset$, if $x_{1} \in \arg \max \left\{f(y) \mid y \leq z_{1}\right\}$, then there exists $x_{2}$ such that

$$
x_{2} \in \arg \max \left\{f(y) \mid y \leq z_{2}\right\}, \quad z_{2} \wedge x_{1} \leq x_{2} .
$$

$\left(-\mathrm{SC}^{2}\right)$ For any $z_{1}, z_{2} \in \mathbf{Z}^{V}$ such that $z_{1} \geq z_{2}$ and $\arg \max \left\{f(y) \mid y \leq z_{1}\right\} \neq \emptyset$, if $x_{2} \in \arg \max \left\{f(y) \mid y \leq z_{2}\right\}$, then there exists $x_{1}$ such that

$$
x_{1} \in \arg \max \left\{f(y) \mid y \leq z_{1}\right\}, \quad z_{2} \wedge x_{1} \leq x_{2} .
$$

\footnotetext{
${ }^{1}$ The original statement in [33] assumes that $\operatorname{dom} f=\{0,1\}^{V}$. However, the proof in [33] is valid for the above case.
} 
The properties $\left(-\mathrm{SC}^{1}\right)$ and $\left(-\mathrm{SC}^{2}\right)$ are interpreted as follows. $\left(-\mathrm{SC}^{1}\right)$ says that when the consumable quota of each commodity decreases or remains the same, the consumer wants a consumption such that the numbers of the commodities whose quotas remain the same do not decrease. $\left(-\mathrm{SC}^{2}\right)$ says that when each quota increases or remains the same, the consumer wants a consumption such that the numbers of the commodities which fail to fill the original quotas do not increase. If $f$ is a set function (i.e., is defined on the hypercube $\left.\{0,1\}^{V}\right)$ then $\left(-\mathrm{SC}^{1}\right)$ and $\left(-\mathrm{SC}^{2}\right)$ are equivalent to conditions of substitutability in Sotomayor [41, Definition 4], and if arg max always gives a singleton (in this case $\left(-\mathrm{SC}^{1}\right)$ and $\left(-\mathrm{SC}^{2}\right)$ coincide) then these are equivalent to persistence (substitutability) in Alkan and Gale [1].

Farooq and Tamura [9] gave characterizations of $\mathrm{M}^{\natural}$-concave set functions by using strengthened properties $\left(-\mathrm{SC}_{\mathrm{G}}^{1}\right)$ and $\left(-\mathrm{SC}_{\mathrm{G}}^{2}\right)$ of $\left(-\mathrm{SC}^{1}\right)$ and $\left(-\mathrm{SC}^{2}\right)$.

$\left(-\mathrm{SC}_{\mathrm{G}}^{1}\right)$ For any $p \in \mathbf{R}^{V}, f[-p]$ satisfies $\left(-\mathrm{SC}^{1}\right)$.

$\left(-\mathrm{SC}_{\mathrm{G}}^{2}\right)$ For any $p \in \mathbf{R}^{V}, f[-p]$ satisfies $\left(-\mathrm{SC}^{2}\right)$.

Theorem 3.5 [9]. For $f:\{0,1\}^{V} \rightarrow \mathbf{R} \cup\{-\infty\}$ with a nonempty effective domain, we have

$$
\left(-\mathrm{M}^{\natural}-\mathrm{EXC}\right) \Leftrightarrow\left(-\mathrm{SC}_{\mathrm{G}}^{1}\right) \Leftrightarrow\left(-\mathrm{SC}_{\mathrm{G}}^{2}\right) .
$$

\section{§4. Overview of Two-Sided Matching Market Models}

In the theory of two-sided matching markets there are two standard models: the stable marriage model due to Gale and Shapley [16] and the assignment model due to Shapley and Shubik [39]. The difference between these two models is that the former does not include money or transferable utilities and the latter permits side payments (see Roth and Sotomayor [37] for details of these models).

In this section, we first explain characterizations of the stability of these two models in terms of utility functions, because this is useful for understanding relations among models based on discrete convex analysis and existing models.

Let $M$ and $W$ denote two disjoint sets of agents and $E$ be the set of all pairs $(i, j)$ of agents $i \in M$ and $j \in W$, i.e., $E=M \times W$. Agents in $M$ or $W$ may be recognized as men or women. For all pairs $(i, j) \in E$, we have pairs $\left(a_{i j}, b_{i j}\right)$. In the assignment model, $a_{i j}$ and $b_{i j}$ are interpreted as profits of $i$ and $j$ when $i$ and $j$ form a partnership. In the stable marriage model, $a_{i j}$ and $b_{i j}$ define preferences as follows: man $i \in M$ prefers $j_{1}$ to $j_{2}$ if $a_{i j_{1}}>a_{i j_{2}}$, and 
$j_{1}$ and $j_{2}$ are indifferent for $i$ if $a_{i j_{1}}=a_{i j_{2}}$ (similarly, preferences of women $j \in W$ are defined by $b_{i j}$ 's). Here, we assume that $a_{i j}>0$ if $j$ is acceptable to $i$, and $a_{i j}=-\infty$ otherwise, and $b_{i j}>0$ if $i$ is acceptable to $j$, and $b_{i j}=-\infty$ otherwise. Define two aggregated utility functions $f_{M}$ for $M$ and $f_{W}$ for $W$ as follows: for all $x \in \mathbf{Z}^{E}$,

(4.1) $f_{M}(x)= \begin{cases}\sum_{(i, j) \in E} a_{i j} x_{i j} & \text { if } x \in\{0,1\}^{E} \text { and } \sum_{j \in W} x_{i j} \leq 1 \text { for all } i \in M \\ -\infty & \text { otherwise, }\end{cases}$

(4.2) $f_{W}(x)= \begin{cases}\sum_{(i, j) \in E} b_{i j} x_{i j} & \text { if } x \in\{0,1\}^{E} \text { and } \sum_{i \in M} x_{i j} \leq 1 \text { for all } j \in W \\ -\infty & \text { otherwise. }\end{cases}$

It is known that functions $f_{M}$ and $f_{W}$ in (4.1) and (4.2) are $\mathrm{M}^{\natural}$-concave.

We now consider one of comprehensive variations of the stable marriage model, in which unacceptability and indifference are allowed. The model deals with the stability of matchings, where a matching is a subset of $E$ such that every agent appears at most once in the subset. Given a matching $X, i \in$ $M$ (respectively $j \in W$ ) is called unmatched in $X$ if there exists no $j \in W$ (resp. $i \in M)$ such that $(i, j) \in X$. A pair $(i, j) \notin X$ is said to be a blocking pair for $X$ if $i$ and $j$ prefer each other to their partners or to being alone in $X$. A matching $X$ is called stable if all pairs $(i, j)$ in $X$ are acceptable for $i$ and $j$, and if there is no blocking pair for $X$. The stability of a matching is characterized as follows. All men $i \in M$ are assigned values $q_{i}$ and all women $j \in W$ are assigned $r_{j}$. A matching $X$ is stable if and only if

(m1) $q_{i}=a_{i j}>-\infty$ and $r_{j}=b_{i j}>-\infty$ for all $(i, j) \in X$,

(m2) $q_{i}=0$ (resp. $r_{j}=0$ ) if $i$ (resp. $j$ ) is unmatched in $X$,

(m3) $q_{i} \geq a_{i j}$ or $r_{j} \geq b_{i j}$ for all $(i, j) \in E$.

The stability can also be characterized by utility functions $f_{M}$ and $f_{W}$ in (4.1) and (4.2). A binary vector $x$ on $E$ is stable in this model ${ }^{2}$ if and only if there exist binary vectors $z_{M}$ and $z_{W}$ such that

$$
\begin{aligned}
& \mathbf{1}=z_{M} \vee z_{W}, \\
& x \text { maximizes } f_{M} \text { in }\left\{y \in \mathbf{Z}^{E} \mid y \leq z_{M}\right\}, \\
& x \text { maximizes } f_{W} \text { in }\left\{y \in \mathbf{Z}^{E} \mid y \leq z_{W}\right\},
\end{aligned}
$$

\footnotetext{
${ }^{2}$ We identify a subset $X$ with its characteristic vector $\chi_{X}$.
} 
where 1 denotes the vector of all ones on $E$. This characterization can be interpreted as follows. We note that $x$ satisfying (4.4) and (4.5) must be a matching because the zero vector attains 0 (finite value) for both $f_{M}$ and $f_{W}$. For a matching $x$, condition (4.4) (resp. (4.5)) claims that each man (resp. woman) selects one of the best partners among admitted partners in $z_{M}$ (resp. $\left.z_{W}\right)$. Therefore, (4.3) guarantees that there is no pair whose members prefer each other to their partners matched in $x$ or to being alone in $x$. Conversely, for a stable matching $x, z_{M}$ can be constructed as follows. Set $z_{M}(i, j)=0$ for all pairs $(i, j) \in E$ such that man $i$ prefers woman $j$ to his partner or to being alone in $x$ (note that by the stability of $x, j$ does not prefer $i$ to her partner or to being alone in $x$ ), and set $z_{M}(i, j)=1$ otherwise. Similarly, $z_{W}$ can be constructed from $x$. Thus, the constraint $y \leq z_{M}$ (resp. $y \leq z_{W}$ ) expresses the situation where each man (resp. woman) cannot establish partnerships with women (resp. men) matched with better men (resp. women).

We next consider the assignment model which includes side payments. An outcome is a triple of payoff vectors $q=\left(q_{i}: i \in M\right) \in \mathbf{R}^{M}, r=\left(r_{j}: j \in W\right) \in$ $\mathbf{R}^{W}$, and a subset $X \subseteq E$, denoted by $(q, r ; X)$. An outcome $(q, r ; X)$ is called stable if

(a1) $X$ is a matching,

(a2) $q_{i}+r_{j}=a_{i j}+b_{i j}$ for all $(i, j) \in X$,

(a3) $q_{i}=0$ (resp. $r_{j}=0$ ) if $i$ (resp. $j$ ) is unmatched in $X$,

(a4) $q \geq \mathbf{0}, r \geq \mathbf{0}$, and $q_{i}+r_{j} \geq a_{i j}+b_{i j}$ for all $(i, j) \in E$,

where $\mathbf{0}$ denotes a zero vector of an appropriate dimension and $p_{i j}\left(=b_{i j}-\right.$ $\left.r_{j}=q_{i}-a_{i j}\right)$ means a side payment from $j$ to $i$ for each $(i, j) \in X$. The stability says that no pair $(i, j) \notin X$ will be better off by making a partnership. Shapley and Shubik [39] proved the existence of stable outcomes by linear programming duality. The maximum weight bipartite matching problem with weights $\left(a_{i j}+b_{i j}\right)$ and its dual problem are formulated by linear programs:

$$
\begin{aligned}
\text { Maximize } & \sum_{(i, j) \in E}\left(a_{i j}+b_{i j}\right) x_{i j} \\
\text { subject to } & \sum_{j \in W} x_{i j} \leq 1 \quad \text { for all } i \in M \\
& \sum_{i \in M} x_{i j} \leq 1 \quad \text { for all } j \in W \\
& x_{i j} \geq 0 \quad \text { for all }(i, j) \in E,
\end{aligned}
$$




$$
\begin{aligned}
& \text { Minimize } \sum_{i \in M} q_{i}+\sum_{j \in W} r_{j} \\
& \text { subject to } q_{i}+r_{j} \geq a_{i j}+b_{i j} \quad \text { for all }(i, j) \in E \\
& \qquad q_{i} \geq 0 \text { for all } i \in M \\
& r_{j} \geq 0 \text { for all } j \in W .
\end{aligned}
$$

Thus, $(q, r ; X)$ is a stable outcome if and only if $x=\chi_{X}, q$ and $r$ are optimal solutions of the above problems, because (a1) and (a4) require the primal and dual feasibility and because (a2) and (a3) mean the complementary slackness. Furthermore, the stability in the assignment model can be characterized by using utility functions in (4.1) and (4.2). A binary vector $x$ on $E$ is $\operatorname{stable}^{3}$ if and only if there exists a real vector $p$ on $E$ such that

$$
\begin{aligned}
& x \text { maximizes } f_{M}[+p], \\
& x \text { maximizes } f_{W}[-p] .
\end{aligned}
$$

This is because a stable outcome $(q, r ; X)$ gives $x=\chi_{X}$ together with $p$ satisfying (4.6) and (4.7) by putting $p_{i j}=b_{i j}-r_{j}$ for all $(i, j) \in E$, and conversely, $x=\chi_{X}$ and $p$ satisfying (4.6) and (4.7) lead us to a stable outcome $(q, r ; X)$ such that $q_{i}=a_{i j}+p_{i j}$ and $r_{j}=b_{i j}-p_{i j}$ for all $(i, j) \in X$ and $q_{i}=0$ (resp. $r_{j}=0$ ) for all $i$ (resp. $j$ ) unmatched in $X$. Conditions (4.6) and (4.7) imply that each agent has one of the best partnerships in $x$ with respect to the utility modified by side payment vector $p$. Moreover, (4.6) and (4.7) say that a stable matching is a competitive equilibrium, and vice versa.

We next briefly introduce relations among models based on discrete convex analysis and related existing models.

Gale and Shapley [16] gave a constructive proof of the existence of a stable matching of the stable marriage model. Since the advent of Gale and Shapley's paper a large number of variations and extensions have been proposed in the literature. Recently, a remarkable extension has been made by Fleiner [10]. He extended the stable marriage model to the framework of matroids and showed the existence of a stable solution. The preference of each person in Fleiner's model can be described by a linear utility function on a matroidal domain. This aspect was extended by Eguchi and Fujishige [6] to the framework of discrete convex analysis. In the Eguchi-Fujishige model, each

\footnotetext{
${ }^{3}$ We say that a binary vector $x$ is stable if and only if there exists a stable outcome $(q, r ; X)$ such that $x$ is the characteristic vector of $X$.
} 
agent can express his/her preference by an $\mathrm{M}^{\natural}$-concave function. Their model is also a concrete example (in terms of utility functions) of the generalized models (in terms of choice functions with substitutability) by Roth [35, 36], Sotomayor [41], Alkan and Gale [1] and Fleiner [11], because an $\mathrm{M}^{\natural}$-concave function defines a choice function with substitutability (see Lemma 3.3). Furthermore, Eguchi, Fujishige and Tamura [7] extended the Eguchi-Fujishige model so that indifference on preferences and multiple partnerships are allowed.

For the other standard model, the assignment model, various extensions have also been proposed since Shapley and Shubik's paper. Sotomayor [42] studies a many-to-many variant of the assignment model, in which each agent can form multiple partnerships with agents of the opposite set without repetition of the same pair, and showed the existence of a stable outcome in the model. Sotomayor [44] also verified the nonemptiness of the core in a manyto-many model with heterogeneous agents, in which repetition of partnerships of each pair is allowed. Kelso and Crawford [21] introduced a many-to-one labor market model in which a utility function of each firm has the gross substitutability and a utility function of each worker is strictly increasing (not necessarily linear) in salary. Danilov, Koshevoy and Murota [5] provided, for the first time, a model based on discrete convex analysis. Danilov, Koshevoy and Lang [4] dealt with a model in which commodities are partitioned into two groups: substitutes and complements.

On the other hand, research has been made toward unifying the stable marriage model and the assignment model. Kaneko [20] gave a general model that includes the two models by means of characteristic functions and proved the nonemptiness of the core. Roth and Sotomayor [38] proposed a general model that encompasses the stable marriage model and the assignment model, and investigated the lattice property for payoffs in their model. However, they did not provide any guarantee for the existence of a stable outcome. Eriksson and Karlander [8] proposed a hybridization of the stable marriage model and the assignment model, for which they verified the existence of a stable outcome. Sotomayor [43] also made further investigation of the hybrid model of Eriksson and Karlander with full generality, and gave a non-constructive proof of the existence of a stable outcome. Fujishige and Tamura [14] generalized the hybrid model due to Eriksson and Karlander [8] and Sotomayor [43], by utilizing $\mathrm{M}^{\natural}$-concave utilities and verified the existence of a stable solution of the general model. Their model includes many models in this section as special cases. 


\section{$\S 5 . \quad$ Arrow-Debreu Type Model}

This section studies an Arrow-Debreu type economy with a finite set $L$ of producers, a finite set $H$ of consumers, a finite set $K$ of indivisible commodities and a perfectly divisible commodity, namely money. Productions of producers and consumptions of consumers are integer-valued vectors in $\mathbf{Z}^{K}$ representing the numbers of indivisible commodities that they produce and consume. Here producers' inputs are represented by negative numbers and their outputs by positive numbers, and conversely, consumers' inputs are represented by positive numbers and their outputs by negative numbers. In the model, for a given price vector $p=(p(k): k \in K) \in \mathbf{R}^{K}$ of commodities, each producer $l$ independently schedules a production in order to maximize $l$ 's profit, and each consumer $h$ independently schedules a consumption to maximize $h$ 's utility under $h$ 's budget constraint, and all agents exchange commodities by buying or selling those through money.

We assume that producer l's profit is described by his/her cost function $C_{l}: \mathbf{Z}^{K} \rightarrow \mathbf{R} \cup\{+\infty\}$ whose value is expressed in units of money. That is, l's profit function $\pi_{l}: \mathbf{R}^{K} \rightarrow \mathbf{R}$ is defined by: for all $p \in \mathbf{R}^{K}$

$$
\pi_{l}(p)=\max _{y \in \mathbf{Z}^{K}}\left\{\langle p, y\rangle-C_{l}(y)\right\} .
$$

Producer l's supply function (correspondence) $S_{l}: \mathbf{R}^{K} \rightarrow 2^{\mathbf{Z}^{K}}$ represents the set of all productions which attain the maximum of $l$ 's profit for a given price vector, that is, for all $p \in \mathbf{R}^{K}$

$$
S_{l}(p)=\arg \max _{y \in \mathbf{Z}^{K}}\left\{\langle p, y\rangle-C_{l}(y)\right\} .
$$

Each consumer $h \in H$ has an initial endowment of indivisible commodities and money which is represented by a vector $\left(x_{h}^{\circ}, m_{h}^{\circ}\right) \in \mathbf{Z}_{+}^{K} \times \mathbf{R}_{+}$, where $\mathbf{Z}_{+}$and $\mathbf{R}_{+}$denote the sets of all nonnegative integers and nonnegative reals, respectively. We note that $x_{h}^{\circ}(k)$ denotes the number of commodities $k \in K$ and $m_{h}^{\circ}$ the amount of money in his/her initial endowment. In the model, each consumer $h$ shares in the profits of the producers and $\theta_{l h}$ denotes the share of the profit of producer $l$ owned by consumer $h$. The numbers $\theta_{l h}$ are nonnegative and $\sum_{h \in H} \theta_{l h}=1$ for all $l \in L$. Thus, consumer $h$ gains an income which is expressed by a function $\beta_{h}: \mathbf{R}^{K} \rightarrow \mathbf{R}$ defined by: for all $p \in \mathbf{R}^{K}$.

$$
\beta_{h}(p)=\left\langle p, x_{h}^{\circ}\right\rangle+m_{h}^{\circ}+\sum_{l \in L} \theta_{l h} \pi_{l}(p) .
$$

We assume that each consumer's utility is quasilinear in money. That is, consumer $h$ 's utility is represented by a quasilinear utility function $\bar{U}_{h}: \mathbf{Z}^{K} \times \mathbf{R} \rightarrow$ 
$\mathbf{R} \cup\{-\infty\}$ defined by: for all $(x, m) \in \mathbf{Z}^{K} \times \mathbf{R}$

$$
\bar{U}_{h}(x, m)=U_{h}(x)+m
$$

where $U_{h}: \mathbf{Z}^{K} \rightarrow \mathbf{R} \cup\{-\infty\}$ whose value is expressed in units of money. It is natural to assume that $\operatorname{dom} U_{h}$ is bounded because none can consume an infinite number of indivisible commodities. We further assume that the amount of money $m_{h}^{\circ}$ in $h$ 's initial endowment is sufficiently large for any $h \in H$. Since consumer $h$ 's schedule maximizes $\bar{U}_{h}$ under the budget constraint, $h$ 's behavior is formulated in terms of an optimization problem

$$
\begin{aligned}
& \text { Maximize } U_{h}(x)+m \\
& \text { subject to }\langle p, x\rangle+m \leq \beta_{h}(p) .
\end{aligned}
$$

Since $\operatorname{dom} U_{h}$ is bounded and $m_{h}^{\circ}$ is large, we can take $m=\beta_{h}(p)-\langle p, x\rangle$ to reduce the above problem to an unconstrained optimization problem

$$
\text { Maximize } U_{h}(x)-\langle p, x\rangle \text {. }
$$

Thus, we can define $h$ 's demand function (correspondence) $D_{h}: \mathbf{R}^{K} \rightarrow 2^{\mathbf{Z}^{K}}$ by: for all $p \in \mathbf{R}^{K}$

$$
D_{h}(p)=\arg \max _{x \in \mathbf{Z}^{K}}\left\{U_{h}(x)-\langle p, x\rangle\right\} .
$$

A tuple $\left(\left(x_{h} \mid h \in H\right),\left(y_{l} \mid l \in L\right), p\right)$, where $x_{h} \in \mathbf{Z}^{K}, y_{l} \in \mathbf{Z}^{K}$ and $p \in \mathbf{R}^{K}$, is called a competitive equilibrium if the following conditions hold:

$$
\begin{aligned}
& x_{h} \in D_{h}(p) \quad(h \in H), \\
& y_{l} \in S_{l}(p) \quad(l \in L), \\
& \sum_{h \in H} x_{h}=\sum_{h \in H} x_{h}^{\circ}+\sum_{l \in L} y_{l}, \\
& p \geq 0 .
\end{aligned}
$$

That is, each agent achieves what he/she wishes to achieve, the balance of supply and demand holds and an equilibrium price vector is nonnegative. The nonnegativity of an equilibrium price vector (5.4) may be ignored in several models in the literature, e.g., in the assignment model (see (4.6) and (4.7)).

A function $U: \mathbf{Z}^{K} \rightarrow \mathbf{R} \cup\{-\infty\}$ is said to be monotone nondecreasing if $x \leq y \Rightarrow U(x) \leq U(y)$ for any $x, y \in \operatorname{dom} U$. Gul and Stacchetti [17] showed the existence of a competitive equilibrium in an exchange economy under the gross substitutability and the monotone nondecreasing condition. 
By Theorem 3.4, we see that Theorem 5.1 is a generalization of the result. Theorems 5.1 and 5.2 stated explicitly by Murota [28, 29] are implied by the results of Danilov, Koshevoy and Murota [5]. We call the model of this section the Danilov-Koshevoy-Murota model or simply the DKM-model if each $C_{l}$ is $\mathrm{M}^{\natural}$-convex and each $U_{h}$ is $\mathrm{M}^{\natural}$-concave.

Theorem 5.1 $[5,28,29]$. In an exchange economy case, where $L=\emptyset$, the DKM-model has a competitive equilibrium $\left(\left(x_{h} \mid h \in H\right), p\right)$ for any initial total endowment $x^{\circ} \in \sum_{h \in H} \operatorname{dom} U_{h}$, where the summation means the Minkowski sum.

Theorem 5.2 [5, 28, 29]. If the continuous version of the DKM-model, which is obtained by regarding all indivisible commodities as divisible, has a competitive equilibrium for an initial total endowment, then the DKM-model also has a competitive equilibrium $\left(\left(x_{h} \mid h \in H\right),\left(y_{l} \mid l \in L\right), p\right)$ of indivisible commodities, where cost functions and utility functions in the continuous model are the convex extensions of $C_{l}$ and concave extensions of $U_{h}$, respectively.

The equilibrium price vectors form a well-behaved polyhedron, $\mathrm{L}^{\natural}$-convex polyhedron investigated by Fujishige and Murota [13] and Murota and Shioura [31]. A polyhedron $P \subseteq \mathbf{R}^{K}$ is called an $L^{\natural}$-convex polyhedron if for all $\alpha$ with $0 \leq \alpha \in \mathbf{R}$

$$
p, q \in P \Longrightarrow(p-\alpha \mathbf{1}) \vee q, p \wedge(q+\alpha \mathbf{1}) \in P .
$$

Theorem $5.3[28,29]$. Suppose that the DKM-model has a competitive equilibrium for an initial total endowment $x^{\circ}$. Then the set $P^{*}\left(x^{\circ}\right)$ of all the equilibrium price vectors is an $L^{\natural}$-convex polyhedron. This means in particular $(\alpha=0$ in (5.5)) that

$$
p, q \in P^{*}\left(x^{\circ}\right) \Longrightarrow p \vee q, p \wedge q \in P^{*}\left(x^{\circ}\right) .
$$

(5.6) implies the existence of the smallest equilibrium price vector, and furthermore, it implies the existence of the largest equilibrium price vector if $P^{*}\left(x^{\circ}\right)$ is bounded.

In order to find a competitive equilibrium, we adopt the aggregate utility function $\Psi^{\prime}: \mathbf{Z}^{K} \rightarrow \mathbf{R} \cup\{ \pm \infty\}$ of the market defined by: for all $z \in \mathbf{Z}^{K}$

$$
\Psi^{\prime}(z)=\sup \left\{-\sum_{l \in L} C_{l}\left(y_{l}\right)+\sum_{h \in H} U_{h}\left(x_{h}\right) \mid \sum_{h \in H} x_{h}-\sum_{l \in L} y_{l}=z\right\} .
$$


We can show that a solution satisfying (5.1), (5.2) and (5.3) attains $\Psi^{\prime}\left(x^{\circ}\right)$ and vice versa. By using the fact that $\Psi^{\prime}$ is the integer convolution of the family of $\mathrm{M}^{\natural}$-concave functions $\left\{-C_{l} \mid l \in L\right\} \cup\left\{U_{h} \mid h \in H\right\}$, Murota and Tamura [34] gave an efficient algorithm for finding a competitive equilibrium. Their algorithm consists of two phases: the first phase computes productions and consumptions satisfying (5.1), (5.2) and (5.3) by solving the $\mathrm{M}^{\natural}$-concave intersection problem, and the second phase finds an equilibrium price vector by solving a shortest path problem.

\section{§6. A Model with Substitutes and Complements}

The DKM-model is regarded as a model consisting of all substitutes. Danilov, Koshevoy and Lang [4] consider an extended model with substitutes and complements, and showed the existence of a competitive equilibrium in the model. In the setting of Section 5, the model assumes

- $K=L \times H$,

- for any producer $l \in L, C_{l}$ is defined on $\{0,1\}^{\{l\} \times H}$ and $y_{l}\left(l^{\prime}, h\right)=0$ is assumed for all $l^{\prime} \in L \backslash\{l\}$ and $h \in H$,

- for any consumer $h \in H, U_{h}$ is defined on $\{0,1\}^{L \times\{h\}}$ and $x_{h}\left(l, h^{\prime}\right)=0$ is assumed for all $l \in L$ and $h^{\prime} \in H \backslash\{h\}$,

- for any consumer $h \in H, x_{h}^{\circ}=\mathbf{0}$,

- a competitive equilibrium is defined by (5.1), (5.2) and (5.3).

A remarkable feature of the model is that $L$ is partitioned into $L_{s}$ and $L_{c}$. They give an assumption called a compatibility principle which imposes the following conditions: (i) $C_{l}$ is $\mathrm{M}^{\natural}$-convex for any $l \in L_{s}$, (ii) $C_{l}$ is submodular for any $l \in L_{c}$ and (iii) $U_{h}$ is a sum of an $\mathrm{M}^{\natural}$-concave set function on $L_{s} \times\{h\}$ and a supermodular set function (i.e., the negative of the submodular set function) on $L_{c} \times\{h\}$ for any $h \in H$. The compatibility principle guarantees the existence of a competitive equilibrium.

Theorem 6.1 [4]. Under the compatibility principle, the above model has a competitive equilibrium. 


\section{§7. Combinatorial Auction}

In this section, we briefly explain a combinatorial auction of non-identical commodities. Let $V$ be a set of commodities for sale by an auctioneer and $B$ a set of buyers. Each buyer $i \in B$ has its private utility function $f_{i}$ in terms of money for all subsets $X$ of $V$ such that $f_{i}(X)$ describes the amount of money paid by buyer $i$ when $i$ gets set $X$ of commodities. The auctioneer's aim is to find an optimal allocation, where an allocation is a subpartition of $V$ into pairwise disjoint subsets $\left\{V_{i} \mid i \in B\right\}$ of $V$ and an optimal allocation is one that maximizes $\sum_{i \in B} f_{i}\left(V_{i}\right)$.

B. Lehmann, D. Lehmann and Nisan [22] discussed combinatorial auctions with several classes of utility functions, and pointed out that if all $f_{i}$ are $\mathrm{M}^{\natural}$ concave then an optimal allocation can be found efficiently as follows. Here we identify a subset with its characteristic vector. Let us consider a function $f_{0}$ which is identically zero on $\{0,1\}^{V}$, which is $\mathrm{M}^{\natural}$-concave. Then, the problem of finding an optimal allocation can be formulated as the problem of finding a maximizer of

$$
f(\mathbf{1})=\max \left\{\sum_{i \in\{0\} \cup B} f_{i}\left(x_{i}\right) \mid \sum_{i \in\{0\} \cup B} x_{i}=\mathbf{1}, x_{i} \in\{0,1\}^{V}(\forall i \in\{0\} \cup B)\right\} .
$$

Since $f$ is the integer convolution of $\mathrm{M}^{\natural}$-concave functions $\left\{f_{i} \mid i \in\{0\} \cup\right.$ $B\}$, the problem can be transformed to the $\mathrm{M}^{\natural}$-concave intersection problem. Furthermore, we have $\operatorname{dom} f_{i}=\{0,1\}^{V}$ for all $i \in\{0\} \cup B$. Thus, the problem is easier than the general case of the $\mathrm{M}^{\natural}$-concave intersection problem. In fact, Murota [24, 27] gave an algorithm for maximizing the sum of two $\mathrm{M}^{\natural}$-concave set functions, whose time complexity is polynomial in $|V|$.

\section{§8. Generalized Stable Marriage Models}

Eguchi and Fujishige [6] proposed a generalized stable marriage model based on discrete convex analysis, and Eguchi, Fujishige and Tamura [7] extend it so that indifference on preferences and multiple partnerships are allowed. We call the model of [7] the EFT-model. In this section, we introduce the EFTmodel.

Let $M$ and $W$ denote two disjoint sets of agents and $E$ be a finite set. In the model, utilities of $M$ and $W$ over $E$ are respectively described by $\mathrm{M}^{\natural}$ concave functions $f_{M}, f_{W}: \mathbf{Z}^{E} \rightarrow \mathbf{R} \cup\{-\infty\}$. Furthermore, we assume that $f_{M}$ and $f_{W}$ satisfy the following assumption. 
(A) Effective domains $\operatorname{dom} f_{M}$ and $\operatorname{dom} f_{W}$ are bounded and hereditary, and have the common minimum point $\mathbf{0}$,

where heredity means that $\mathbf{0} \leq x_{1} \leq x_{2} \in \operatorname{dom} f_{M}$ (resp. $\left.\operatorname{dom} f_{W}\right)$ implies $x_{1} \in \operatorname{dom} f_{M}\left(\right.$ resp. $\left.\operatorname{dom} f_{W}\right)$. The heredity of effective domains implies that each agent can arbitrarily decrease the multiplicity of partnerships without any agreement of the partner, similarly as in other two-sided matching market models.

Let $z$ be an integral vector such that

$$
\operatorname{dom} f_{M} \cup \operatorname{dom} f_{W} \subseteq\left\{y \in \mathbf{Z}^{E} \mid \mathbf{0} \leq y \leq z\right\} .
$$

Taking conditions $(4.3) \sim(4.5)$ into account, we say that $x \in \operatorname{dom} f_{M} \cap \operatorname{dom} f_{W}$ is an $f_{M} f_{W}$-stable solution if there exist $z_{M}, z_{W} \in \mathbf{Z}^{E}$ such that

$$
\begin{aligned}
& z=z_{M} \vee z_{W}, \\
& x \in \arg \max \left\{f_{M}(y) \mid y \leq z_{M}\right\}, \\
& x \in \arg \max \left\{f_{W}(y) \mid y \leq z_{W}\right\} .
\end{aligned}
$$

Condition (8.2) replaces the upper bound vector 1 in (4.3) by $z$. Since functions in (4.1) and (4.2) are $\mathrm{M}^{\natural}$-concave, the model includes the stable marriage model as a special case. For the case where $f_{M}$ and $f_{W}$ are defined by (4.1) and (4.2), conditions (8.2), (8.3) and (8.4) claim that there is no pair in $E$ whose members prefer each other to their partners matched in $x$ or to being alone in $x$.

Eguchi, Fujishige and Tamura showed the existence of an $f_{M} f_{W}$-stable solution.

Theorem 8.1 [7]. For any $M^{\natural}$-concave functions $f_{M}, f_{W}: \mathbf{Z}^{E} \rightarrow \mathbf{R} \cup$ $\{-\infty\}$ satisfying $(\mathrm{A})$, the EFT-model always has an $f_{M} f_{W}$-stable solution.

Theorem 8.1 can be shown constructively by a generalization of the GaleShapley algorithm [16] as follows. To describe the algorithm, we assume that we are initially given $x_{M}, x_{W} \in \mathbf{Z}^{E}$ and $z_{M}, z_{W} \in \mathbf{Z}^{E}$ satisfying (8.2) and the following:

$$
\begin{aligned}
& x_{M} \in \arg \max \left\{f_{M}(y) \mid y \leq z_{M}\right\}, \\
& x_{W} \in \arg \max \left\{f_{W}(y) \mid y \leq z_{W} \vee x_{M}\right\}, \\
& x_{W} \leq x_{M} .
\end{aligned}
$$

We can easily compute such initial vectors by setting $z_{M}=z, z_{W}=\mathbf{0}$, and by finding $x_{M}$ and $x_{W}$ such that

$$
\begin{aligned}
& x_{M} \in \arg \max \left\{f_{M}(y) \mid y \leq z_{M}\right\}, \\
& x_{W} \in \arg \max \left\{f_{W}(y) \mid y \leq x_{M}\right\} .
\end{aligned}
$$


The algorithm is described as follows.

$\operatorname{Algorithm~G\_ GS~}\left(f_{M}, f_{W}, x_{M}, x_{W}, z_{M}, z_{W}\right)$

Input: $M^{\natural}$-concave functions $f_{M}, f_{W}$ and $x_{M}, x_{W}, z_{M}, z_{W}$ satisfying (8.2), (8.5), (8.6), (8.7).

Step 1. Find an element $x_{M}$ in $\arg \max \left\{f_{M}(y) \mid x_{W} \leq y \leq z_{M}\right\}$.

Step 2. Find an element $x_{W}$ in $\arg \max \left\{f_{W}(y) \mid y \leq x_{M}\right\}$.

Step 3. For all $e \in E$ with $x_{M}(e)>x_{W}(e)$, set $z_{M}(e):=x_{W}(e), z_{W}(e):=z(e)$.

Step 4. If $x_{M}=x_{W}$ then output $\left(x_{M}, x_{W}, z_{M}, z_{W} \vee x_{M}\right)$. Else go to Step 1.

It should be noted here that because of Assumption (A), $x_{M}$ and $x_{W}$ are well-defined within the effective domains and that algorithm G_GS terminates after at most $\sum_{e \in E} z(e)$ iterations, because $\sum_{e \in E} z_{M}(e)$ is strictly decreased in each iteration. We finally give an outline of a proof of Theorem 8.1.

Outline of Proof of Theorem 8.1. Let $x_{M}^{(i)}, x_{W}^{(i)}, z_{M}^{(i)}$, and $z_{W}^{(i)}$ be $x_{M}, x_{W}$, $z_{M}$, and $z_{W}$ obtained after the $i$ th iteration in G_GS for $i=1,2, \cdots, t$, where $t$ is the last to get the outputs. By Lemma 3.3, we can show that for all $i=1, \cdots, t$

$$
\begin{aligned}
& x_{M}^{(i+1)} \in \arg \max \left\{f_{M}(y) \mid y \leq z_{M}^{(i)}\right\}, \\
& x_{W}^{(i)} \in \arg \max \left\{f_{W}(y) \mid y \leq z_{W}^{(i)} \vee x_{M}^{(i)}\right\} .
\end{aligned}
$$

Thus we have for $i=t$

$$
\begin{aligned}
& x_{M}^{(t)} \in \arg \max \left\{f_{M}(y) \mid y \leq z_{M}^{(t)}\right\}, \\
& x_{W}^{(t)} \in \arg \max \left\{f_{W}(y) \mid y \leq z_{W}^{(t)} \vee x_{M}^{(t)}\right\}, \\
& x_{M}^{(t)}=x_{W}^{(t)} .
\end{aligned}
$$

By the way of modifying $z_{M}, z_{W}$, and $x_{M}$ we have

$$
z_{M}^{(t)} \vee\left(z_{W}^{(t)} \vee x_{M}^{(t)}\right)=z .
$$

This is an outline of the proof of Theorem 8.1. 


\section{§9. A Generalized Hybridization}

Fujishige and Tamura [14] proposed a generalized hybridization of the stable marriage model and the assignment model, by extending the idea of Eriksson and Karlander [8] and the models in [6, 7].

In this model, utilities (in monetary terms) of $M$ and $W$ are respectively described by $\mathrm{M}^{\natural}$-concave functions $f_{M}, f_{W}: \mathbf{Z}^{E} \rightarrow \mathbf{R} \cup\{-\infty\}$ satisfying Assumption (A), and, in addition, $E$ is partitioned into two subsets $F$ (the set of flexible elements) and $R$ (the set of rigid elements). In the hybrid model due to Eriksson and Karlander [8], $M$ and $W$ are, respectively, partitioned into $\left\{M_{F}, M_{R}\right\}$ and $\left\{W_{F}, W_{R}\right\}$, and we have $F=M_{F} \times W_{F}$ and $R=E \backslash F$, where $E=M \times W$.

Let $z$ be an integral vector satisfying (8.1). For a vector $d$ on $E$ and $S \subseteq E$, we assume that $\left.d\right|_{S}$ denotes the restriction of $d$ on $S$. We say that $x \in \operatorname{dom} f_{M} \cap \operatorname{dom} f_{W}$ is an $f_{M} f_{W}$-stable solution with respect to $(F, R)$ if there exist $p \in \mathbf{R}^{E}$ and $z_{M}, z_{W} \in \mathbf{Z}^{R}$ such that

$$
\begin{aligned}
\left.p\right|_{R} & =\mathbf{0}, \\
\left.z\right|_{R} & =z_{M} \vee z_{W}, \\
x & \in \arg \max \left\{f_{M}[+p](y)|y|_{R} \leq z_{M}\right\}, \\
x & \in \arg \max \left\{f_{W}[-p](y)|y|_{R} \leq z_{W}\right\} .
\end{aligned}
$$

Condition (9.1) states that there are no side payments for all rigid elements. Obviously, if $E=R$ then our model includes the Eguchi-Fujishige-Tamura model, and if $E=F$ then it includes the assignment model because functions in (4.1) and (4.2) are $\mathrm{M}^{\natural}$-concave (see also (4.6) and (4.7)).

The main result of [14] is the following.

Theorem 9.1 [14]. For any $M^{\natural}$-concave functions $f_{M}, f_{W}: \mathbf{Z}^{E} \rightarrow \mathbf{R} \cup$ $\{-\infty\}$ satisfying $(\mathrm{A})$ and for any partition $(F, R)$ of $E$, there always exists an $f_{M} f_{W}$-stable solution with respect to $(F, R)$.

In [14], Theorem 9.1 is shown constructively by combining algorithm G_GS in Section 8 and a successive shortest path algorithm for maximizing $f_{M}+f_{W}$, which is a modified version of that in [23].

\section{$\S 10 . \quad$ Concluding Remarks}

We finally discuss open problems.

Algorithm G_GS in Section 8 solves the maximization problem of an $\mathrm{M}^{\natural}$ concave function in each iteration. As we mentioned in Section 2, it is known 
that a maximizer of an $\mathrm{M}^{\natural}$-concave function $f$ on $E$ can be found in polynomial time in $n$ and $\log L$, where $n=|E|$ and $L=\max \left\{|| x-y \|_{\infty} \mid x, y \in \operatorname{dom} f\right\}$. Since G_GS terminates after at most $\sum_{e \in E} z(e)$ iterations, the time complexity of G_GS is $\mathrm{O}(\operatorname{poly}(n) \cdot L)$, where $L=\|z\|_{\infty}$. Unfortunately, there exist a series of examples in which G_GS requires numbers of iterations proportional to $L$. For the special case where $f_{M}$ and $f_{W}$ are linear on rectangular effective domains, Baïou and Balinski [2] showed that an $f_{M} f_{W}$-stable solution of the EFT-model can be found in polynomial time in $n$. However, it is open whether an $f_{M} f_{W}$-stable solution for the general case can be found in polynomial time in $n$ and $\log L$.

Open Problem 1. Develop a polynomial time algorithm for finding an $f_{M} f_{W}$-stable solution of the EFT-model.

We note that the problem of checking whether a given point $x \in \operatorname{dom} f_{M} \cap$ $\operatorname{dom} f_{W}$ is $f_{M} f_{W}$-stable in the EFT-model can be solved in $\mathrm{O}\left(n^{2}\right)$ time by using the following local criterion.

Lemma 10.1 [7]. A point $x \in \operatorname{dom} f_{M} \cap \operatorname{dom} f_{W}$ is $f_{M} f_{W}$-stable in the EFT-model if and only if it satisfies the following conditions:

$$
\begin{aligned}
\text { for each } e \in E, f_{M}(x) & \geq f_{M}\left(x-\chi_{e}\right) \quad \text { and } \quad f_{W}(x) \geq f_{W}\left(x-\chi_{e}\right), \\
\text { for each } e \in E, f_{M}(x) & \geq f_{M}\left(x+\chi_{e}-\chi_{e^{\prime}}\right) \quad\left(\forall e^{\prime} \in E \cup\{0\}\right) \quad \text { or } \\
f_{W}(x) \geq f_{W}\left(x+\chi_{e}-\chi_{e^{\prime \prime}}\right) & \left(\forall e^{\prime \prime} \in E \cup\{0\}\right) .
\end{aligned}
$$

Since the model in Section 9 (the FT-model) is an extension of the EFTmodel, we also propose the next open problem.

Open Problem 2. Develop a polynomial time algorithm for finding an $f_{M} f_{W}$-stable solution with respect to an arbitrary partition $(F, R)$ of $E$.

We note that for the case where $f_{M}$ and $f_{W}$ are integer-valued functions and $E=F$, we can find an $f_{M} f_{W}$-stable solution with respect to partition $(E, \emptyset)$ efficiently, because the problem can be transformed to the $\mathrm{M}^{\natural}$-concave intersection problem.

The stability of the FT-model seems to be rather technical. We hope that there exist a natural common generalization of the stable marriage model and the assignment model, which also includes the FT-model as a special case.

Open Problem 3. $\quad$ Propose a natural model including the stable marriage model, the assignment model and the FT-model. 


\section{References}

[1] Alkan, A. and Gale, D., Stable schedule matching under revealed preference, J. Econom. Theory, 112 (2003), 289-306.

[2] Baïou, M. and Balinski, M., Erratum: The stable allocation (or ordinal transportation) problem, Math. Oper. Res., 27 (2002), 662-680.

[3] Danilov, V., Koshevoy, G. and Lang, C., Gross substitution, discrete convexity, and submodularity, Discrete Appl. Math., 131 (2003), 283-298.

[4] Substitutes, complements and equilibrium in two-sided market models, in: Sertel, M. R. and Koray, S., eds., Advances in Economic Design, (Springer-Verlag, Berlin, 2003), 105-125.

[5] Danilov, V., Koshevoy, G. and Murota, K., Discrete convexity and equilibria in economies with indivisible goods and money, Math. Social Sci., 41 (2001), 251-273.

[6] Eguchi, A. and Fujishige, S., An extension of the Gale-Shapley matching algorithm to a pair of $\mathrm{M}^{\natural}$-concave functions, Discrete Mathematics and Systems Science Research Report 02-05, Osaka University, 2002.

[7] Eguchi, A., Fujishige, S. and Tamura, A., A generalized Gale-Shapley algorithm for a discrete-concave stable-marriage model, in: Ibaraki, T., Katoh, N. and Ono, H., eds., Proceedings of the 14th International Symposium ISAAC2003, LNCS, 2906 (SpringerVerlag, Berlin, 2003), 495-504.

[8] Eriksson, K. and Karlander, J., Stable matching in a common generalization of the marriage and assignment models, Discrete Math., 217 (2000), 135-156.

[9] Farooq, R. and Tamura, A., A new characterization of $\mathrm{M}^{\natural}$-convex set functions by substitutability, J. Oper. Res. Soc. Japan, 47 (2004), 18-24.

[10] Fleiner, T., A matroid generalization of the stable matching polytope, in: Gerards, B. and Aardal, K., eds., Proceedings of the 8th International IPCO Conference, LNCS, 2081 (Springer-Verlag, Berlin, 2001), 105-114.

[11] A fixed point approach to stable matchings and some applications, Math. Oper. Res., 28 (2003), 103-126.

[12] Fujishige, S., Submodular Functions and Optimization, Ann. Discrete Math. 47, NorthHolland, Amsterdam, 1991.

[13] Fujishige, S. and Murota, K., Notes on L-/M-convex functions and the separation theorems, Math. Program., 88 (2000), 129-146.

[14] Fujishige, S. and Tamura, A., A general two-sided matching market with discrete concave utility functions, RIMS Preprints No. 1401, Kyoto University, 2003.

[15] Fujishige, S. and Yang, Z., A note on Kelso and Crawford's gross substitutes condition, Math. Oper. Res., 28 (2003), 463-469.

[16] Gale, D. and Shapley, L. S., College admissions and the stability of marriage, Amer. Math. Monthly, 69 (1962), 9-15.

[17] Gul, F. and Stacchetti, F., Walrasian equilibrium with gross substitutes, J. Econom. Theory, 87 (1999), 95-124.

[18] Iwata, S., Moriguchi, S. and Murota, K., Capacity scaling algorithm for M-convex submodular flow, in: Bienstoc, D. and Nemhauser, G., eds., Integer Programming and Combinatorial Optimization: 10th International IPCO Conference, LNCS, 3064 (SpringerVerlag, Berlin, 2004), 352-367.

[19] Iwata, S. and Shigeno, M., Conjugate scaling algorithm for Fenchel-type duality in discrete convex optimization, SIAM J. Optim., 13 (2002), 204-211.

[20] Kaneko, M., The central assignment game and the assignment markets, J. Math. Econom., 10 (1982), 205-232.

[21] Kelso, Jr. A. S. and Crawford, V. P., Job matching, coalition formation, and gross substitutes, Econometrica, 50 (1982), 1483-1504.

[22] Lehmann, B., Lehmann, D. and Nisan, N., Combinatorial auctions with decreasing marginal utilities, Games Econom. Behav., to appear.

[23] Moriguchi, S. and Murota, K., Capacity scaling algorithm for scalable M-convex submodular flow problems, Optim. Methods Softw., 18 (2003), 207-218. 
[24] Murota, K., Valuated matroid intersection, II: Algorithms, SIAM J. Discrete Math., 9 (1996), 562-576.

[25] - Convexity and Steinitz's exchange property, Adv. Math., 124 (1996), 272-311.

[26] Discrete convex analysis, Math. Program., 83 (1998), 313-371.

[27] _ Matrices and Matroids for Systems Analysis, Springer-Verlag, Berlin, 2000.

[28] _ Discrete Convex Analysis — An Introduction (in Japanese), Kyoritsu Publishing Co., Tokyo, 2001.

[29] - Discrete Convex Analysis, Society for Industrial and Applied Mathematics, Philadelphia, 2003.

[30] Murota, K. and Shioura, A., M-convex function on generalized polymatroid, Math. Oper. Res., 24 (1999), 95-105.

[31] Extension of M-convexity and L-convexity to polyhedral convex functions, Adv. in Appl. Math., 25 (2000), 352-427.

[32] $\_$, Relationship of M-/L-convex functions with discrete convex functions by Miller and by Favati-Tardella, Discrete Appl. Math., 115 (2001), 151-176.

[33] Murota, K. and Tamura, A., New characterizations of M-convex functions and their applications to economic equilibrium models with indivisibilities, Discrete Appl. Math., 131 (2003), 495-512.

[34] Application of M-convex submodular flow problem to mathematical economics, Japan J. Indust. Appl. Math., 20 (2003), 257-277.

[35] Roth, A. E., Stability and polarization of interests in job matching, Econometrica, 52 (1984), 47-57.

[36] Conflict and coincidence of interest in job matching: Some new results an open questions, Math. Oper. Res., 10 (1985), 379-389.

[37] Roth, A. E. and Sotomayor, M. A. O., Two-Sided Matching - A Study in GameTheoretic Modeling and Analysis, Cambridge University Press, Cambridge, 1990.

[38] Stable outcomes in discrete and continuous models of two-sided matching: A unified treatment, Rev. Econom., 16 (1996), 1-24.

[39] Shapley, L. S. and Shubik, M., The assignment game I: The core, Internat. J. Game Theory, 1 (1972), 111-130.

[40] Shioura, A., Fast scaling algorithms for M-convex function minimization with application to the resource allocation problem, Discrete Appl. Math., 134 (2004), 303-316.

[41] Sotomayor, M., Three remarks on the many-to-many stable matching problem: Dedicated to David Gale on his 75th birthday, Math. Social Sci., 38 (1999), 55-70.

[42] - The lattice structure of the set of stable outcomes of the multiple partners assignment game, Internat. J. Game Theory, 28 (1999), 567-583.

[43] _ Existence of stable outcomes and the lattice property for a unified matching market, Math. Social Sci., 39 (2000), 119-132.

[44] A labor market with heterogeneous firms and workers, Internat. J. Game Theory, 31 (2002), 269-283.

[45] Tamura, A., Coordinatewise domain scaling algorithm for M-convex function minimization, Math. Program., to appear. 